\title{
POWER BROADENING AND NONLINEAR FIR MAGNETO-PHOTOCONDUCTIVITY IN n-GaAs
}

\author{
W. Prettl \\ Institut für Angewandte Physik der Universität Regensburg \\ $D-8400$ Regensburg, W-Germany
}

and

\begin{abstract}
A. Vass, G. R. Allan, and C. R. Pidgeon
Heriot-Watt University, Edinburgh, Scotland
\end{abstract}

Received March 25, 1983

The saturation of the photoconductivity due to $1 s-2 p_{+}$ shallow donor transitions in n-GaAs has been investigated using a high power cw-FIR-laser. Magnetic field strengths were chosen in such a way that $2 \mathrm{p}+$ energy levels were either below or above the $\mathrm{N}=0$ conduction band Landau level. In the former case $1 \mathrm{~s}-2 \mathrm{p}_{+}$transitions are found to be inhomogeneously broadened with a saturation intensity as low as $0.84 \mathrm{~mW} / \mathrm{cm}^{2}$, giving an effective lifetime of the $2 \mathrm{p}+$ state of $1.5 \mu \mathrm{s}$. Above the band edge the integrated photoconductivity does not saturate though the intensity-normalized peak photosignal decreases and the linewidth increases with raising intensity. This strange behaviour is tentatively attributed to optical excitations of $2 \mathrm{p}_{+}$electrons to higher 1ying electron Landau states.

Key word: semiconductors, epitaxial GaAs, shallow donors, nonlinear photoconductivity, power broadening. 


\section{Introduction}

High power far-infrared (FIR) lasers enable the application of nonlinear spectroscopic methods in the long wavelength infrared spectral range. Several investigations of nonlinear optical phenomena including the saturation of cyclotron resonance and impurity transitions in semiconductors $[1,2,3]$, two photon absorption [4], and second harmonic generation [5] have been performed, using TEA laserpumped systems. In a previous paper we reported on cw FIR nonlinear magnetoabsorption of $1 \mathrm{~s}-2 \mathrm{p}_{+}$shallow donor transitions in high purity epitaxial n-GaAs [6]. Saturation intensities ten times lower than deduced from earlier pulsed laser experiments [3] and correspondingly long lifetimes of the $2 \mathrm{p}+$ state were observed, showing that it is essential to use cw lasers for these studies. Further dramatic changes of the $2 p+$ state 1 ifetime could be attributed to a new effect, the optical magnetoimpurity resonance (OMR).

In the present work we give an account of the nonlinear photoconductivity due to $1 \mathrm{~s}-2 \mathrm{p}_{+}$donor transitions. As in the case of absorption, the saturation behaviour was found to change drastically with magnetic field. For the $2 p_{+}$state below the conduction band the intensity dependence of the resonant photoconductive signal can be described with high accuracy in terms of an inhomogeneously broadened line, allowing the measurement of the $2 p_{+}$state lifetime. In the case of the $2 \mathrm{p}+$ state lying in the conduction band no saturation of the integrated normalized photoconductivity could be observed within the available FIR intensity range. This behaviour must result from additional optical excitations of electrons from the upper state, probably in connection with the OMR, whose details cannot be determined from the present experiments.

The intensity dependence of the photoconductivity is discussed in terms of a three level model taking into account the $1 \mathrm{~s}$ ground state, the $2 \mathrm{p}_{+}$state and the $\mathrm{N}=0$ Landau subband. The effective lifetime of the $2 p_{+}$state is evaluated in terms of the $2 p_{+}$to $1 \mathrm{~s}$ phonon transition lifetime, the $2 \mathrm{p}_{+}$to conduction band transfer rate, and the conduction band to $1 \mathrm{~s}$ ground state 1 ifetime. 


\section{Experimental Technique}

The nonlinear photoconductivity was measured in an epitaxial layer of $59 \mu \mathrm{m}$ thickness having a donor concentration of $\mathrm{N}_{\mathrm{D}}=2.76 \cdot 10^{14} \mathrm{~cm}^{-3}$ and a rather large compensation ratio $\mathrm{K}=\mathrm{N}_{\mathrm{A}} / \mathrm{N}_{\mathrm{D}}=0.85$, where $\mathrm{N}_{\mathrm{A}}$ is the density of acceptors. The sample was mounted in a metallic light pipe at the center of a superconducting solenoid and immersed in liquid helium at $4.2 \mathrm{~K}$. The measurements were performed in the Faraday configuration with the electric field perpendicular to the magnetic field. A high power Edinburgh Instruments optically pumped FIR laser, operated either $\mathrm{cw}$ or with controlled pulse lengths, was applied using $\mathrm{CH}_{3} \mathrm{OH}$ laser lines. In pulsed measurements the pulse duration was adjusted to be longer than any expected relaxation time ensuring steady state conditions during optical excitation. The FIR intensity in the sample was determined by a calibrated pyroelectric detector taking into account the losses of the light pipe and the reflection at the crystal surface. Due to light pipe optics the sample was subjected to unpolarized radiation. Therefore the effective intensity was one half of the total intensity in the sample, because circular polarized radiation only is absorbed in Faraday configuration.

The photoconductivity was recorded by standard lock-in techniques applying a fast response circuit. The bias voltage was taken in the ohmic range of the current voltage characteristic, avoiding impact ionization nonlinearities [7]. In all measurements the same bias voltage was applied in order to obtain comparable quantitative results.

\section{Experimental Results}

The $2 p+$ level of shallow donors in GaAs crosses the $N=0$ conduction band Landau level at a magnetic fieid sightly below $B=2 \mathrm{~T}[8]$. The photoconductivity was measured as a function of the magnetic field strength for various FIR intensities at three different wavelengths $\lambda=186 \mu \mathrm{m}, 164 \mu \mathrm{m}$ and $152 \mu \mathrm{m}$ corresponding to the $2 \mathrm{p}_{+}$ level being below the conduction band, slightly above level crossing, and wel1 in the lowest Landau subband, respectively. The $1 \mathrm{~s}-2 \mathrm{p}_{+}$transitions occuring for these wavelengths at $B=1.4 \mathrm{~T}, 2.0 \mathrm{~T}$ and $2.4 \mathrm{~T}$, respectively, are shown in Fig. 1 together with computed donor and conduction band Landau levels [8]. 


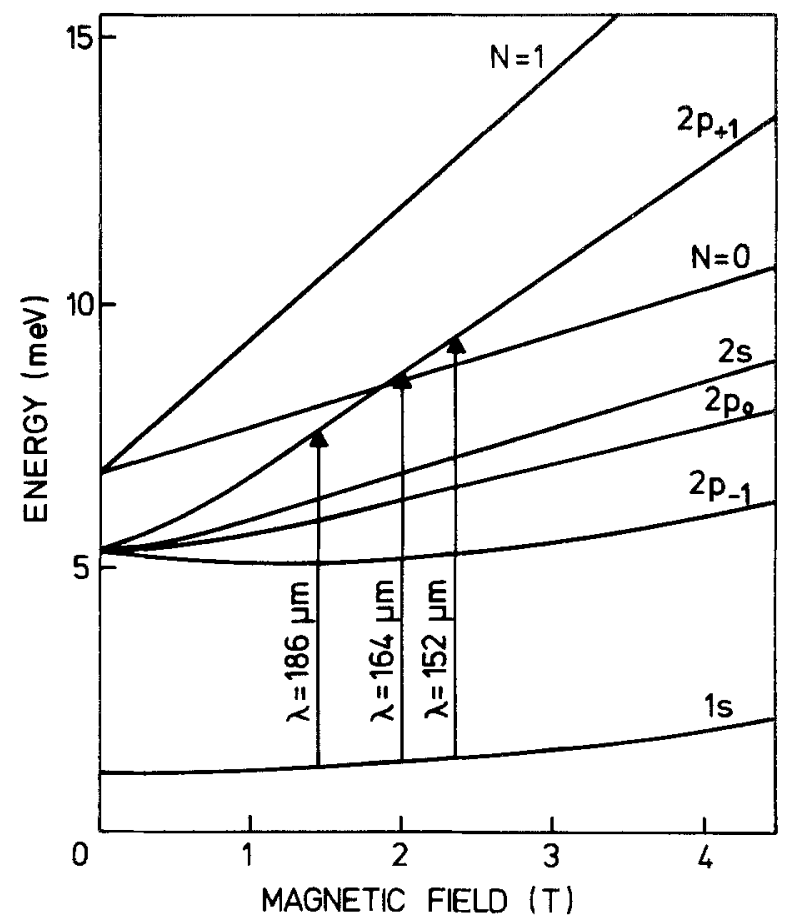

Figure 1. Magnetic fields dependence of shallow donor states and Landau levels (after Ref. 8) and $1 \mathrm{~s}-2 \mathrm{p}+$ transitions measured at three different photon energies.

Photoconductivity at low intensities varies linearly with the intensity $I$. Therefore saturation effects are most clearly illustrated by considering the photoconductive signal divided by the intensity, which will be called normalized photoconductivity. A typical measurement of the relative normalized photoconductivity, $\Delta \sigma / \sigma \mathrm{I}$, at $\lambda=164 \mu \mathrm{m}$ is plotted in Fig. 2 for three different intensities, showing the saturation of the resonant signal. The excitation of $2 p_{+}$states is observed as a narrow 1 ine on a continuous background, which did not saturate within the applied intensity range. The background signal is assumed to be due to photoionization of thermally excited electron states. The normalized peak photo voltage, $\Delta \mathrm{V} / \mathrm{I}$, is shown in $\mathrm{Fig} .3$ as a function of intensity. The most drastic saturation of the peak signal is found at $\lambda=186 \mu \mathrm{m}$ where the $2 \mathrm{p}+1$ eve 1 is below the conduction band. For all three wavelengths and all applied intensities the line shape of the resonant signal 


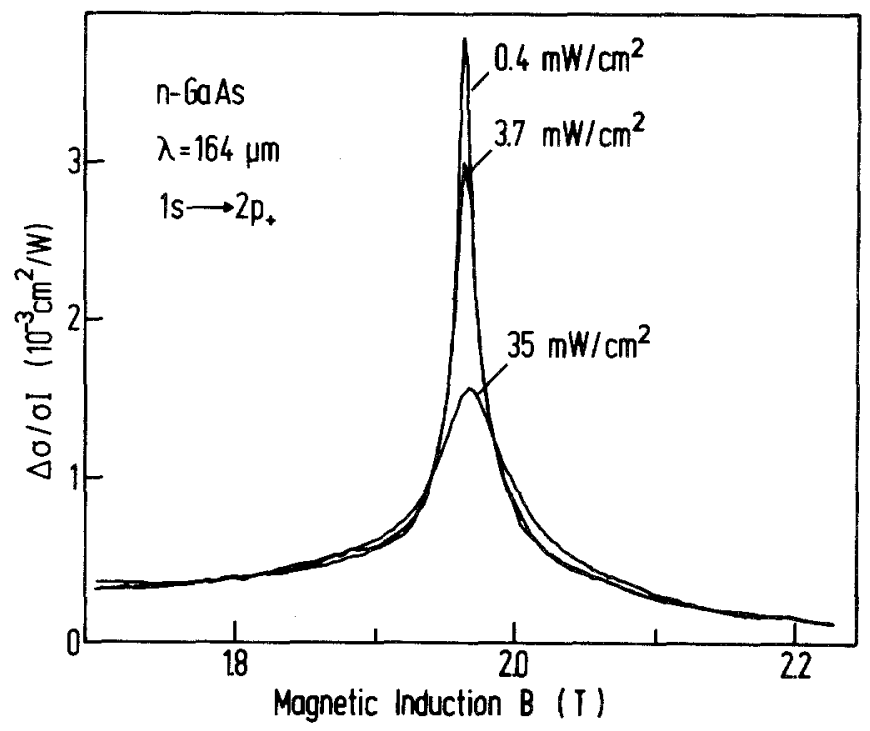

Figure 2. Relative normalized photoconductivity due to $1 \mathrm{~s}-2 \mathrm{p}_{+}$transitions as a function of the magnetic field for three different FIR intensities at $\lambda=164 \mu \mathrm{m}$.

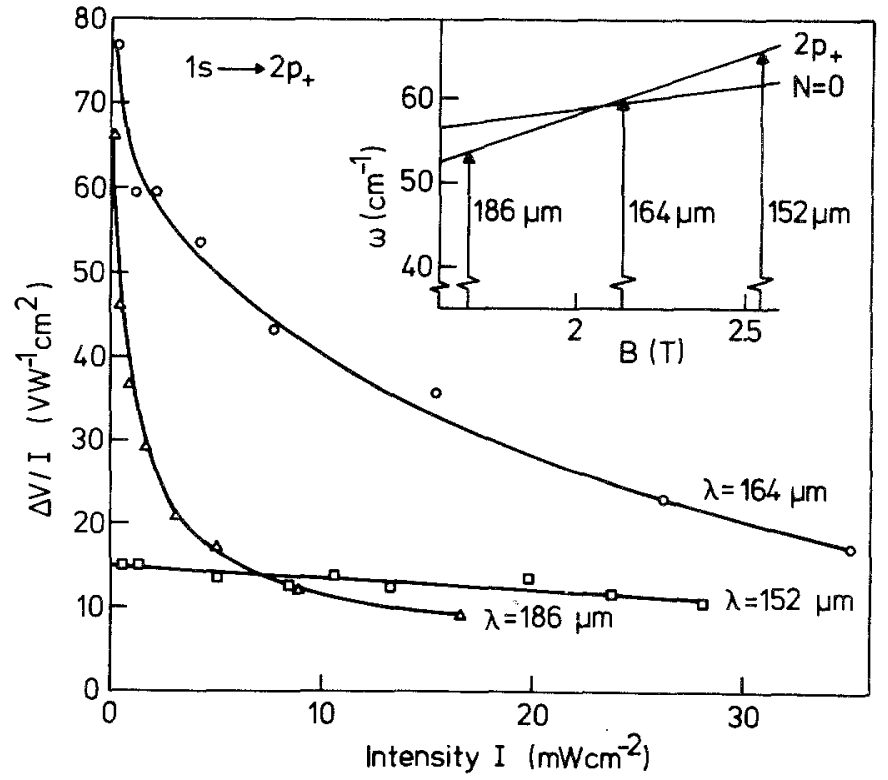

Figure 3. Normalized peak photoconductive voltage, $\Delta V / I$, as a function of intensity I for three different wavelengths $(\triangle V$ after $40 \mathrm{~dB}$ amplification). The insert shows the optical transitions with respect to the involved energy levels. 


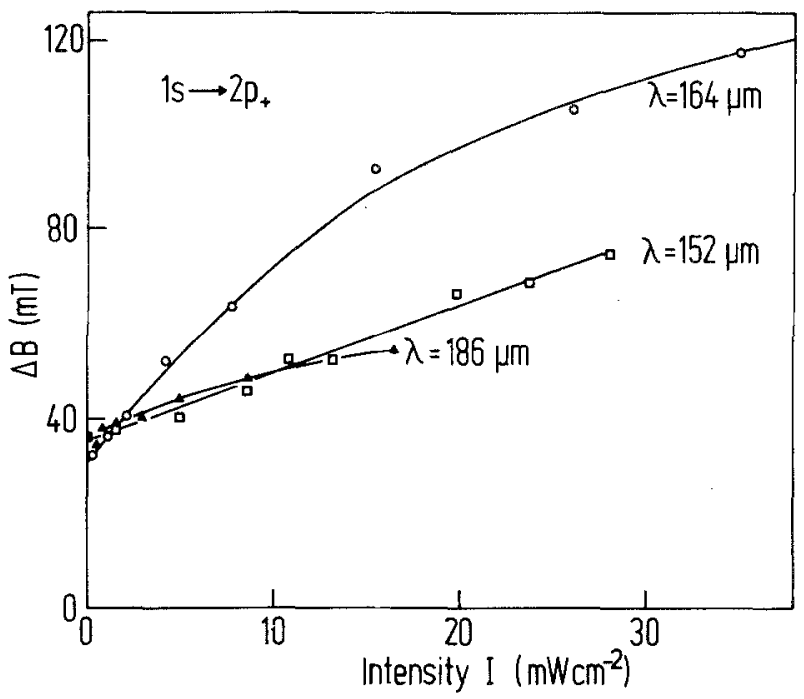

Figure 4. Linewidth of the $1 \mathrm{~s}-2 \mathrm{p}_{+}$photoconductive resonance as a function of intensity for three wavelengths.

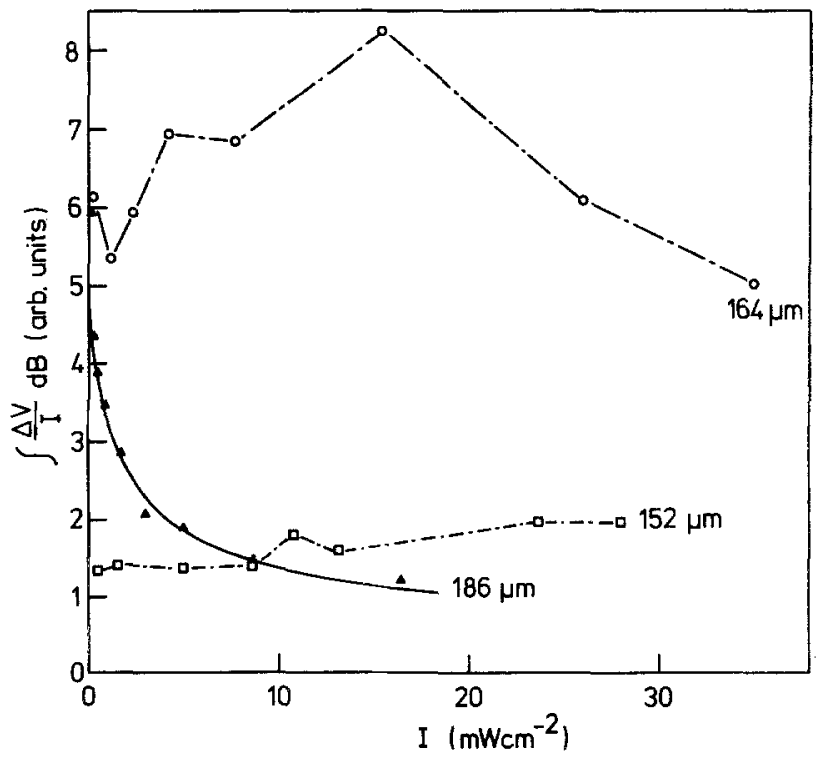

Figure 5. Integrated normalized photoconductivity $\mathrm{I}^{-1} \int \Delta \mathrm{VdB}$ versus intensity. The full 1 ine for $186 \mathrm{\mu m}$ is calculated, the broken lines for $\lambda=164 \mu \mathrm{m}$ and $152 \mu \mathrm{m}$ are only guide lines for the eye. 
was very close to a Lorentzian curve. The measured full width at half maximum height above the background $(\Delta B)$ is plotted as a function of intensity in Fig. 4. The linewidth increases most strongly at $\lambda=164 \mu \mathrm{m} i . e$. where the $2 \mathrm{p}_{+}$state is very close to level crossing; it is more than tripled at the highest available intensity. In Fig. 5 the integrated normalized photoconductive signal $\mathrm{I}^{-1} \int \Delta \mathrm{V} d B$ is displayed versus intensity. This plot shows the important result that true saturation of the photoconductivity occurs at $\lambda=186 \mu \mathrm{m}$ only. In the cases of the $2 p_{+}$level being in the band $(\lambda=164 \mu \mathrm{m}$ and $152 \mu \mathrm{m})$ the decrease of the normalized peak signal with intensity is more or less compensated by the broadening of the line.

\section{Theory and Discussion}

The observed linewidth at low intensities is of the order of $\Delta B=30 \mathrm{mT}$. Because the energy separation $\mathrm{E}_{2 \mathrm{p}_{+}}-\mathrm{E}_{1 \mathrm{~s}}$ depends linearly on the magnetic field strength $B$ in the applied range of $B$, having a slope of $\mathrm{d}\left(\mathrm{E}_{2 \mathrm{p}}-\mathrm{E}_{1 \mathrm{~s}}\right) / \mathrm{dB} \Delta 13 \mathrm{~cm}^{-1} \mathrm{~T}^{-1}[9]$, the magnetic field scale may be converted into a wave number scale giving $\Delta \omega=$ $0.39 \mathrm{~cm}^{-1}$. This width corresponds to the typical central cell splitting of shallow donors in GaAs $[10,11,12]$ showing that the sample under investigation contained predominantly one chemical donor species only.

Shallow impurity transition lines in highly compensated semiconductors are inhomogeneously broadened due to the electric field of ionized donors and acceptors. According to the theory of Larsen [13] randomly distributed charged impurities may lead to Lorentzian line shapes as observed in the present investigation, if the energy level shifts are mainly caused by a quadrupole interaction. The inhomogeneous 1 inewidth $\Delta \omega_{i}$ is proportional to the concentration of charged impurities $N, \Delta \omega_{i} \propto N$. Optical and thermal ionization of neutral donors raises $\mathrm{N}$ and thus increases $\Delta \omega_{i}$. $N$ may rise from its low temperature value $N=2 N_{A}$ up to $\mathrm{N}=\mathrm{N}_{\mathrm{D}}+\mathrm{N}_{\mathrm{A}}$ if all donors are ionized. In the present sample of compensation ratio $\mathrm{K}=0.89$ the linewidth can broaden due to this mechanism at most by a factor 1.1 which is too small to explain the measured large linewidth increase. In other work line broadening of excited state transitions by about a factor of 2 was observed on raising the temperature from $4.2 \mathrm{~K}$ to $10 \mathrm{~K}$. This large effect was 
explained by taking into account the variation of the spatial correlation of ionized donors and acceptors due to temperature dependent phonon-assisted hopping of electrons from neutral to ionized donors [14]. We estimated the heating of our sample assuming that the total laser power is absorbed and the thermal energy loss is achieved by ballistic phonon transport to the crystal surfaces. Therewith we found that even for the highest applied intensity the temperature rises by less than $1 \mathrm{~K}$, which is again too small to affect measurably the linewidth. Thus we conclude that in our measurements power broadening of the homogeneous width due to depletion of the ground state population must be the main reason for the observed line broadening.

The saturation characteristics of the resonant photoconductivity will be discussed on the basis of a three level mode1 $[1,2]$ schematically outlined in Fig. 6 for the case of $\lambda=186 \mu \mathrm{m}$ where the $2 \mathrm{p}_{+}$energy level is below the conduction band. An electron excited into the $2 \mathrm{p}_{+}$state (rate $\mathrm{X}_{\mathrm{O}}$ ) may either relax directly into the $1 \mathrm{~s}$ ground state (rate $\mathrm{T}_{\mathrm{o}}$ ), or can be transferred into the conduction. band (rate $\mathrm{X}_{1}$ ) and then be trapped by an ionized donor (rate $\mathrm{T}_{1}$ times the concentration of positive donors $\mathrm{p}_{\mathrm{D}}$ ). The rate equations are given by

$$
\begin{aligned}
& \mathrm{dn} / \mathrm{dt}=\mathrm{x}_{1} \mathrm{n}_{\mathrm{D}}^{*}-\mathrm{n}_{1} \mathrm{~T}_{1} \mathrm{p}_{\mathrm{D}} \\
& \mathrm{dn}_{\mathrm{D}}^{*} / \mathrm{dt}=\mathrm{x}_{0} \mathrm{n}_{\mathrm{D}}-\mathrm{T}_{0} \mathrm{n}_{\mathrm{D}}^{*}-\mathrm{x}_{1} \mathrm{n}_{\mathrm{D}}^{*} \\
& \mathrm{~d} \mathrm{n}_{\mathrm{D}} / \mathrm{dt}=-\mathrm{x}_{0} \mathrm{n}_{\mathrm{D}}+\mathrm{T}_{0} \mathrm{n}_{\mathrm{D}}^{*}+\mathrm{n}_{1} \mathrm{p}_{\mathrm{D}}
\end{aligned}
$$

Figure 6. Schematic diagram of $1 \mathrm{~s}, 2 \mathrm{p}_{+}$, and $\mathrm{N}=0$ Landau subband three-level system at $1.4 \mathrm{~T}$ corresponding to $\lambda=$ $186 \mu \mathrm{m}$. Rate constants are described in the text.

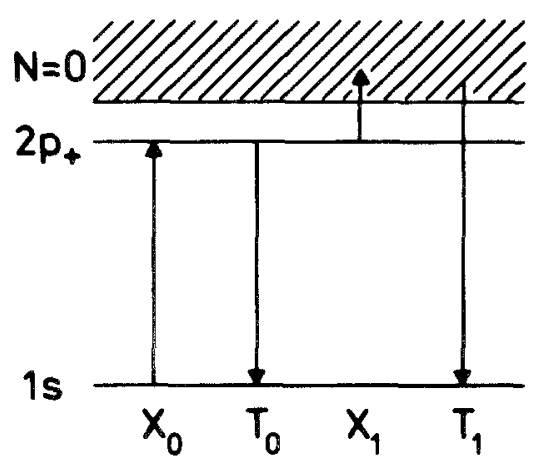


where $n, n_{D}^{*}, n_{D}$ and $p_{D}$ are the concentrations of electrons in the conduction band, ls ground state, $2 p_{+}$excited state and ionized donors. At low temperature ignoring thermal excitation $X_{0}$ is given by $X_{O}=\sigma(\omega) I / h \omega$, where $\sigma(\omega)$, I and $\hbar \omega$ are the optical cross-section, radiation intensity and photon energy. We assume $\sigma(\omega)$ to be

$$
\sigma(\omega)=\frac{\pi}{2} \quad \sigma_{0} \Delta \omega_{h} S_{\Delta \omega_{h}}\left(\omega-\omega_{0}\right) .
$$

Here $\sigma_{0}$ and $\Delta \omega_{h}$ is the peak cross-section and the homogeneous width, respectively, and

$$
S_{\Delta \omega_{h}}\left(\omega-\omega_{0}\right)=\frac{1}{2 \pi} \frac{\Delta \omega_{h}}{\left(\omega-\omega_{0}\right)^{2}+\left(\frac{\Delta \omega_{h}}{2}\right)^{2}}
$$

is a Lorentzian of halfwidth $\Delta \omega_{h}$ being centered at $\omega_{0}$ corresponding to the $2 \mathrm{p}_{+}^{-1} \mathrm{~s}$ energy difference. The relaxation constant $T_{0}$ may be written as $T_{0}=\widetilde{T}_{O}+\sigma(\omega) I / \dot{h} \omega$, where the first term refers to thermal relaxation and the second term to stimulated emission.

Under steady state conditions, taking into account the conservation of total donor number $N_{D}=n_{D}+n_{D}^{*}+p_{D}$ and considering local neutrality $\mathrm{P}_{\mathrm{A}}=\mathrm{N}_{\mathrm{D}}-\mathrm{N}_{\mathrm{A}}=\mathrm{n}_{\mathrm{D}}+\mathrm{n}_{\mathrm{D}}^{*}+\mathrm{n}$, Eqs. (1)-(3) can be solved. The free carrier concentration is determined by:

$$
\mathrm{nT}_{1}\left(\mathrm{~N}_{\mathrm{A}}+\mathrm{n}\right)=\frac{\mathrm{X}_{0} \mathrm{x}_{1}\left(\mathrm{P}_{\mathrm{A}}-\mathrm{n}\right)}{\mathrm{T}_{0}+\mathrm{X}_{0}+\mathrm{x}_{1}} \text {. }
$$

At low temperature in the linear limit, where $\mathrm{n}<<\mathrm{N}_{\mathrm{A}}$, the photoexcited electron concentration divided by the intensity $\mathrm{n} / \mathrm{I}$, being proportional to the normalized photoconductivity, is found to be

$$
\frac{n(\omega)}{I}=\frac{n P_{A} \sigma(\omega)}{1+\tau_{\text {eff }} 2 \sigma(\omega) I / \hbar \omega}
$$

with $n \quad=x_{1}\left\{\hbar \omega T_{1} N_{A}\left(\widetilde{T}_{0}+x_{1}\right)\right\}^{-1}$ and 
(8)

$$
\tau_{\text {eff }}=\frac{\mathrm{x}_{1}+2 \mathrm{~T}_{1} \mathrm{~N}_{A}}{2 \mathrm{~T}_{1} \mathrm{~N}_{A}\left(\tilde{\mathrm{T}}_{0}+\mathrm{X}_{1}\right)}
$$

the effective Iifetime of the $2 p_{+}$state. Using Eqs. (4) and (5), $\mathrm{n} / \mathrm{I}$ in $\mathrm{Eq}$. (7) may be rewritten as

$$
\frac{\mathrm{n}(\omega)}{\mathrm{I}}=\eta \mathrm{P}_{\mathrm{A}} \sigma_{\mathrm{O}} \Delta \Omega\left(1+\frac{\mathrm{I}}{\mathrm{I}_{\mathrm{S}}}\right)^{-1} \frac{\pi}{2} \mathrm{~S}_{\Delta \Omega}\left(\omega-\omega_{\mathrm{O}}\right)
$$

where $I_{s}=\hbar \omega_{0}\left(\tau_{\text {eff }}^{2 \sigma_{0}}\right)^{-1}$ is the saturation intensity and $\mathrm{S}_{\Delta \Omega}$ is a Lorentzian of halfwidth $\Delta \Omega=\Delta \omega_{\mathrm{h}}\left(1+\frac{\mathrm{I}}{\mathrm{I}_{\mathrm{S}}}\right)^{1 / 2}$.

This result shows that linearization of Eq. (6) simply reduces the three leve1 model to an effective two level system of upper state lifetime $\tau_{\text {eff }}$.

Taking into account the inhomogeneous broadening we must fold $\mathrm{n} / \mathrm{I}$ by the distribution of frequencies $\omega_{0}$ which itself is assumed to be of Lorentzian form of halfwidth $\Delta \omega_{i}$. Owing to the fact that folding of two Lorentzian functions reproduces a Lorentzian of added halfwidths, we finally find for $\mathrm{n} / \mathrm{I}$ the same expression as given in Eq. (9) except that $\Delta \Omega$ is replaced by $\Delta \widetilde{\Omega}$ with

$$
\Delta \widetilde{\Omega}=\Delta \omega_{i}+\Delta \Omega=\Delta \omega_{i}+\Delta w_{h}\left(1+\frac{I}{I_{s}}\right)^{1 / 2}
$$

Then the peak normalized electron concentration is given by:

$$
\frac{n\left(\omega_{0}\right)}{I}=\frac{n P_{A} \sigma_{0}}{\left(1+\frac{I}{I_{s}}\right)^{1 / 2}} \cdot \frac{\Delta \omega_{h}}{\Delta \widetilde{\Omega}}
$$

and the integrated normalized electron concentration is found to be

$$
I^{-1} \rho_{n}(\omega) \mathrm{d} \omega=\eta P_{A} \sigma_{0}\left(1+\frac{I}{I_{s}}\right)^{-1 / 2} \frac{\pi}{2} \Delta \omega_{h}
$$

having the expected intensity dependence of an inhomogeneously broadened line. 
Using the last three equations the observed saturation at $\lambda=186 \mu \mathrm{m}$ could be fitted by taking $\Delta \omega_{i}=0.39 \mathrm{~cm}^{-1} \triangleq$ $30 \mathrm{mT}, \Delta \omega_{h}=0.07 \mathrm{~cm}^{-1} \Delta 5.4 \mathrm{mT}$ and $\mathrm{I}_{\mathrm{s}}=0.84 \mathrm{~mW} \mathrm{~cm} \mathrm{~cm}^{-2}$. The excellent agreement with the experimental results is demonstrated in Fig. 5 and Figs. 7 and 8 where the calculated intensity dependence of the normalized peak photosignal and the 1 inewidth are plotted, respectively, together with the measured values.

The optical peak cross-section $\sigma_{o}$ at $\lambda=186 \mu \mathrm{m}$ was determined by transmission measurements [6] to be $\sigma_{0}=$ $4.1 \cdot 10^{-13} \mathrm{~cm}^{2}$. This allows the evaluation of the effective lifetime $\tau_{\text {eff }}=\hbar \omega_{0}\left(I_{s} 2 \sigma_{o}\right)^{-1}$ yielding $\tau_{\text {eff }}=1.5 \mu$ s being about two orders of magnitude longer than obtained in previous pulsed laser experiments [3]. To account for the significance of this result we note that the $2 \mathrm{p}_{+}$to $1 \mathrm{~s}$ phonon transition 1 ifetime $\left(\widetilde{T}_{0}\right)^{-1}$ was derived to be of the order of $10 \mu \mathrm{s}[15,3]$. On the other hand it was shown by measuring the temperature dependence of the photoconductivity that at $4.2 \mathrm{~K}$ and at $\mathrm{B}=0$ almost al1 $2 \mathrm{p}$ electrons are thermally excited to the conduction band, hence $\mathrm{X}_{1} \gg \widetilde{T}_{0}[16]$. Assuming this relation to be valid also at a finite magnetic field strength, $\tau$ fef defined in Eq. (8) may be approximated by $\tau_{\text {eff }} \simeq\left(\mathrm{X}_{1}\right)^{-1}+\left(2 \mathrm{~T}_{1} \mathrm{~N}_{\mathrm{A}}\right)^{-1}$. Thus $\tau_{\text {eff }}$ as measured by the present experiment gives an upper limit of the low temperature conduction band to ground state lifetime $\left(\mathrm{T}_{1} \mathrm{~N}_{\mathrm{A}}\right)^{-1}$. This quantity is dependent on the acceptor concentration and may vary in a wide range. It is mainly determined by the $2 \mathrm{~s}-1 \mathrm{~s}$ thermal transition rate. Comparably long lifetimes as found in the present investigation were observed by $\mathrm{F}$. Brown et al. in transient high power photoconductivity of n-GaAs [17].

The experimental results at $\lambda=164 \mu \mathrm{m}$ and $152 \mu \mathrm{m}$, where the $2 \mathrm{p}_{+}$level is in the $\mathrm{N}=0$ Landau band, are in complete contrast to the clear situation at $\lambda=186 \mu \mathrm{m}$. The intensity dependences of the measured quantities cannot be fitted by the model discussed above. Even at low intensity, where the linear approximation of Eq.(6) certainly holds, no consistent description is possible. As the integral photoconductivity does not saturate we suspect that an additional optical excitation of $2 p_{+}$electrons occurs. This would formally give an intensity dependent rate coefficient $\mathrm{x}_{1}$ raising the photosignal and decreasing $\tau_{\text {eff. }}$. We point out that the energy separation of the $\mathrm{N}=2$ Landau level and the 


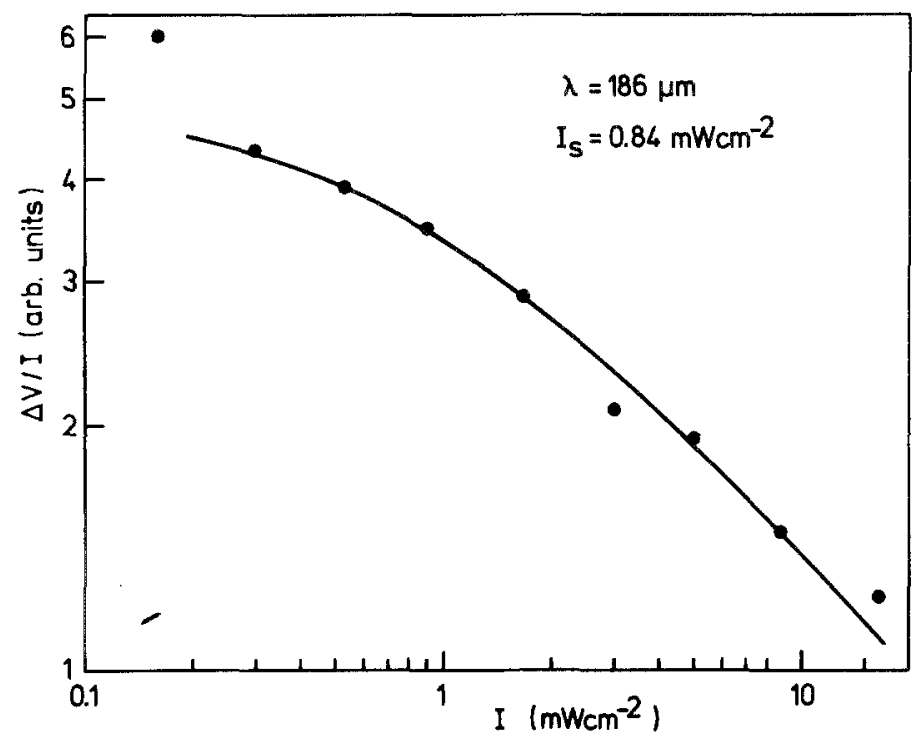

Figure 7. Calculated intensity dependence of the normalized peak photosignal $\lambda=186 \mu \mathrm{m}$ and experimenta1 points.

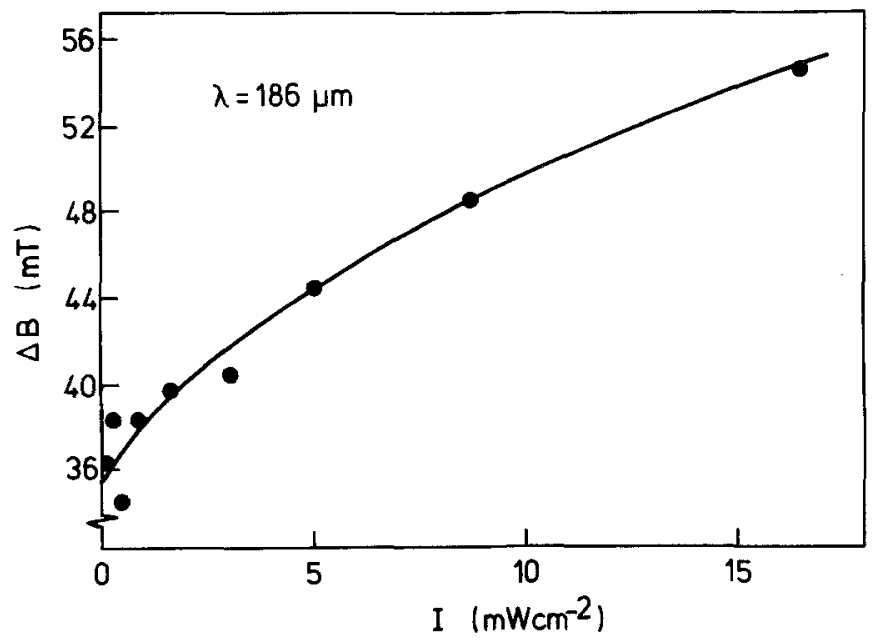

Figure 8. Calculated 1inewidth at $\lambda=186 \mu \mathrm{m}$ as function of intensity and experimental points. 
$2 \mathrm{p}+$ state closely approaches the photon energy with increasing magnetic field strength above $1.4 \mathrm{~T}(\lambda=186 \mu \mathrm{m})$. Hence optical transitions from the $2 p_{+}$level to impurity states connected with the $\mathrm{N}=2$ Landau subband may raise $\mathrm{X}_{1}$ with intensity. In addition at $\lambda=152 \mu \mathrm{m}$ corresponding $\mathrm{B}=2.4 \mathrm{~T}$ the $2 \mathrm{p}_{-}-1 \mathrm{~s}$ magnetoimpurity resonance occurs exciting free carriers into the $\mathrm{N}=1$ Landau level. Both processes contribute to a hot electron population in increasing the electron mobility. Therefore resonant photoconductivity may also be caused by altering the mobility. Such excitation most strongly affects the strength of the photosignal which is dependent on the scattering and relaxation times of free carriers whereas the linewidth might still be a reasonable probe of the upper state Iifetime. Thus we infer from the measured line broadening shown in Fig. 5 that the $2 p_{+}$state lives longest at $\mathrm{B}=2.0 \mathrm{~T}(\lambda=164 \mu \mathrm{m})$. This conclusion precisely agrees with the results of previously performed nonlinear magnetoabsorption measurements [6]. The long 1 ifetime at $164 \mu \mathrm{m}$ in contrast to the other two wavelengths is consistantly explained by the fact that the $2 p_{-}-1$ s transition is far off any magnetoimpurity resonance.

\section{Acknowledgements}

We are very grateful to E. Bauser, Max-Planck-Institut für Festkörperforschung, Stuttgart, for provision of n-GaAs samples, and to R.L. Aggarwad, W.R. Böhm, K.J. Button, E. Gornik, and R.A. Stradling for helpful discussions and suggestions. Two of us (A.V. and G.R.A.) have benefitted from SERC studentships, and one of us (W.P.) thanks S.D. Smith for kind hospitality at Heriot-Watt University and gratefully acknowledges financial support by the Deutsche Forschungsgemeinschaft.

\section{$\underline{\text { References }}$}

1. T. Murotani and Y. Nisida, J.Phys.Soc. Japan 32, 986 (1972).

2. E. Gornik, T.Y. Chang, T.J. Bridges, V.T. Nguyen, I.D. McGee, and W. Mül1er, Phys.Rev.Letters 40, 1151 (1978).

3. K. Muro, N. Yutani, and Sh. Narita, J.Phys.Soc. Japan 49, 593 (1980). 
4. W. Böhm, E. Ettlinger, and W. Prettl, Phys.Rev. Letters 47, $1198(1981)$.

5. F. Keilmann, 7th Int. Conf. on Infrared and Millimeter Waves, Marseille 1983.

6. C.R. Pidgeon, A. Vass, G.R. Allan, W. Prett1, and L.A. Eaves, to be published, Phys.Rev. Letters 1983.

7. E. Schö11, W. Heisel, and W. Prettl, Z.Physik B 47, 287 (1982).

8. D.M. Larsen, J.Phys.Chem. Solids 29, 271 (1968).

9. R. Kaplan, M.A. Kinch, and W.C. Scott, Solid State Commun. $\underline{7}, 883$ (1969).

10. R.A. Stradling, L. Eaves, R.A. Hoult, N. Miura, P.E. Simmonds, and C.C. Bradley, GaAs and Related Compounds (The Institute of Physics, London, 1973), pp. 65.

11. M.N. Afsar, K.J. Button, and G.L. McCoy, Int.J. of Infrared and Mil1imeter Waves 1, 513 (1980).

12. M.N. Afsar, K.J. Button, A.Y. Cho, and H. Morkoc, Int. J. of Infrared and Mil1imeter Waves 2, 1113 (1981).

13. D.M. Larsen, Phys.Rev. B 13, 1681 (1976).

14. J. Golka, J. Trylski, M.S. Skolnick, R.A. Stradling, and Y. Couder, Solid State Commun. 22, 623 (1977).

15. G. Ascare11i and S. Rodriguez, Phys.Rev. 124, 1321 (1961).

16. M. Miyao and Sh. Narita, J.Phys.Soc. Japan 42,128 (1977).

17. F. Brown, A. Adamson, and P.A. Wolff, Int.J. of Infrared and Millimeter Waves 1, 277 (1980). 